\title{
MULTIPLICITY OF A NOETHERIAN INTERSECTION
}

\author{
Andrei Gabrielov and Askold Khovanski
}

October 28, 1997

To Vladimir Igorevich Arnol'd on his 60th birthday

\begin{abstract}
A differential ring of analytic functions in several complex variables is called a ring of Noetherian functions if it is finitely generated as a ring and contains the ring of all polynomials. In this paper, we give an effective bound on the multiplicity of an isolated solution of a system of $n$ equations $f_{i}=0$ where $f_{i}$ belong to a ring of Noetherian functions in $n$ complex variables. In the one-dimensional case, such an estimate is known and has applications in number theory and control theory. Multi-dimensional case presented in this paper provides a solution of a rather old problem concerning finiteness properties of transcendental functions defined by algebraic partial differential equations.
\end{abstract}

\section{INTRODUCTION}

1. The main result. A ring $K$ of analytic functions in an open domain $U \subset \mathbb{C}^{n}$ is called a ring of Noetherian functions in $U$ if

1) $K$ contains the ring $\mathbb{C}\left[x_{1}, \ldots, x_{n}\right]$ of polynomials and is finitely generated over that ring;

2) $K$ is closed under differentiation. In other words, for each $f \in K$, all partial derivatives $\partial f / \partial x_{i}$ belong to $K$. In particular, $K$ is a Noetherian ring, which is the origin of the notation "Noetherian function" introduced by Tougeron [T].

A set of $m$ functions $\psi=\left\{\psi_{1}, \ldots, \psi_{m}\right\}$ is called a Noetherian chain of order $m$ if these functions generate $K$ over $\mathbb{C}\left[x_{1}, \ldots, x_{n}\right]$. A function $\phi$ in $K$ is called a Noetherian function of degree $\beta$ relative to a Noetherian chain $\psi$ if there exists a polynomial $P$ of degree not exceeding $\beta$ in $n+m$ variables such that $\phi=P(x, \psi(x))$. The Noetherian chain $\psi$ has degree not exceeding $\alpha$ if each partial derivative $\partial \psi_{i} / \partial x_{j}$ is a Noetherian function of degree $\alpha$ relative to $\psi$.

Standard Noetherian arguments allow one to prove that the multiplicity of an isolated intersection of Noetherian functions is bounded by a certain function of discrete parameters $n, m, \alpha$, and $\beta$. As usual, these arguments do not provide any effective method of computation of such function. This computation is done in the present paper. The main result of this paper is the following

Partially supported by NSF Grant \# DMS-9704745 and by NSERC Grant \# OGP0156833. Part of this work was done when both authors participated in the program on Singularity Theory and Geometry at the Fields Institute for Research in Mathematical Sciences, Toronto, Canada. 
Theorem 1. Let $\phi_{1}, \ldots, \phi_{n}$ belong to a ring $K$ of Noetherian in $U \subset \mathbb{C}^{n}$. Suppose that all $\phi_{i}$ have degree not exceeding $\beta$ relative to a common Noetherian chain $\psi$ of order $m$ and degree $\alpha \geq 1$. Then the multiplicity of any isolated solution of the system of equations $\phi_{1}=\cdots=\phi_{n}=0$ does not exceed maximum of the following two numbers:

$$
\begin{aligned}
& \frac{1}{2} Q\left((m+1)(\alpha-1)[2 \alpha(n+m+2)-2 m-2]^{2 m+2}+2 \alpha(n+2)-2\right)^{2(m+n)} \\
& \frac{1}{2} Q\left(2(Q+n)^{n}(\beta+Q(\alpha-1))\right)^{2(m+n)}, \quad \text { where } Q=e n\left(\frac{e(n+m)}{\sqrt{n}}\right)^{\ln n+1}\left(\frac{n}{e^{2}}\right)^{n} .
\end{aligned}
$$

2. Pfaff systems with polynomial coefficients. Rings of Noetherian functions and Noetherian chains can be defined in terms of systems of Pfaff equations. This approach is more geometric, and will be used in the proof of the main result.

Definition 1. An analytic $n$-dimensional distribution in an open domain $U \subset \mathbb{C}^{n+m}$ is defined by

$$
d z_{i}=\sum_{j=1}^{n} g_{i j}(x, z) d x_{j}, \quad \text { for } \quad i=1, \ldots, m
$$

where $x \in \mathbb{C}^{n}, z \in \mathbb{C}^{m}$, and $g_{i j}$ are analytic functions in $U$. An integral manifold of an analytic distribution (1) is a $n$-dimensional submanifold $\Lambda \subset U$ tangent to (1), i.e.,

$$
\left.\left(d z_{i}-\sum_{j=1}^{n} g_{i j}(x, z) d x_{j}\right)\right|_{\Lambda} \equiv 0, \quad \text { for } \quad i=1, \ldots, m
$$

Locally, an integral manifold can be represented as a graph of an analytic vector-function $z=\psi(x)=\left(\psi_{1}(x), \ldots, \psi_{m}(x)\right)$ satisfying

$$
\frac{\partial \psi_{i}}{\partial x_{j}}=g_{i j}(x, \psi(x)), \quad \text { for } \quad i=1, \ldots, m \quad \text { and } \quad j=1, \ldots, n \text {. }
$$

A Noetherian chain of order $m$ and degree $\alpha$ is an analytic function $\psi(x)$ satisfying (2) where $g_{i j}(x, z)$ are polynomials in $(x, z) \in \mathbb{C}^{n+m}$ of degree not exceeding $\alpha \geq 1$. A Noetherian function of degree $\beta$ relative to a Noetherian chain $\psi$ is an analytic function $\phi(x)=P(x, \psi(x))$, where $P(x, z)$ is a polynomial in $(x, z)$ of degree not exceeding $\beta$.

3. Theory of fewnomials and local conjectures. Suppose that the system (2) of Pfaff equations is triangular, i.e., that functions $g_{i j}(x, \psi)$ depend only on $x$ and $\psi_{1}, \ldots, \psi_{i}$ (but do not depend on $\psi_{i+1}, \ldots, \psi_{m}$ ). Suppose, in addition, that all functions $\psi_{i}$ and polynomials $g_{i j}$ are real. In this case, $\psi$ is called a Pfaffian chain, and Noetherian functions $P(x, \psi(x))$ where $P$ is a real polynomial are called Pfaffian functions relative to $\psi$. Real solutions of systems of equations with Pfaffian functions have global finiteness properties resembling the finiteness properties of real algebraic sets [Kh1]. These systems, studied in [Kh2, Kh3], have many applications in real algebraic geometry, computational complexity, 
control theory, and model theory. For these finiteness properties, both requirements (that system (2) is triangular and all functions are real) are essential. For example, the simplest Noetherian functions sin and cos have infinite number of real zeros, and a simple Pfaffian function $\exp x-1$ has infinite number of complex zeros.

In the beginning of the 80-ies, Khovanskii conjectured that local finiteness properties remain valid for non-triangular systems, also in the complex domain. Here are three variants of this conjecture, in decreasing order of generality.

Let $\phi_{1}(x, a), \ldots, \phi_{l}(x, a)$ be a set of $l$ analytic functions in a vicinity of a point $\left(x_{0}, a_{0}\right) \in$ $\mathbb{C}^{n+k}$. Suppose that, for each value of parameters $a$, functions $\phi_{i}(x, a)$, considered as analytic functions in $x$, belong to a ring $K$ of Noetherian functions. Let us fix a Noetherian chain of order $m$ and degree $\alpha$ in $K$, and let $\phi_{1}(., a), \ldots \phi_{l}(., a)$ be Noetherian functions of degree not exceeding $\beta$ relative to this Noetherian chain, for all values of $a$.

Conjecture 1. There exists an explicit function $F(n, m, \alpha, \beta, l)$ with the following property. For any small positive $\epsilon$, there exists a positive $\delta$ such that, for any fixed value of a with $\left|a-a_{0}\right|<\delta$, the sum of Betti numbers of the set

$$
\phi_{1}(x, a)=\cdots=\phi_{l}(x, a)=0,\left|x-x_{0}\right|<\epsilon
$$

does not exceed $F(n, m, \alpha, \beta, l)$.

Conjecture 2. Let $\phi_{1}(., a)=\cdots=\phi_{n}(., a)=0$ be a system of $n$ equations in $n$ variables depending on parameters a. Suppose that, for a fixed value of a, functions $\phi_{i}$ are Noetherian of degree not exceeding $\beta$ relative to a Noetherian chain of order $m$ and degree $\alpha$. The number of isolated solutions of this system converging to $x_{0}$ as $a \rightarrow a_{0}$ can be effectively estimated from above in terms of $n, m, \alpha, \beta$. Note that $x_{0}$ can be a non-isolated solution of $\phi_{1}\left(., \alpha_{0}\right)=\cdots=\phi_{n}\left(., a_{0}\right)=0$.

Conjecture 3. Multiplicity of an isolated solution of a system of equations with Noetherian functions with a common Noetherian chain can be effectively estimated from above in terms of the number of variables, order and degree of the Noetherian chain, and degrees of the Noetherian functions.

Theorem 1 of this paper proves Conjecture 3. Conjectures 1 and 2 remain open problems. In fact these two conjectures are equivalent. For Pfaffian functions (triangular systems (2), also in the complex domain) proof of Conjecture 2 was given in [G1]. For this paper, it is important that Conjectures 2 and 3 are true in one-dimensional case.

One-dimensional case. The problem in one-dimensional case was first formulated and solved by Nesterenko [N]. His motivation came from number theory, and his results have important applications in this area (see [W]). Later this problem was re-discovered by Risler $[\mathrm{R}]$, in connection with non-holonomic dynamics and control theory. He was interested in degree of nonholonomy of control systems. Solution of this problem was given by Gabrielov [G2]. Later, Gabrielov found a new solution [G3], more simple and with a better estimate. In this paper, we use bounds on degree of nonholonomy from [G2] and [G3]. Gabrielov's solution was further simplified by Khovanskii, using integration over Euler characteristics. This made solution so simple that it could be generalized to several variables. This generalization is presented in this paper. 
To prove Theorem 1, we need four preliminary steps:

(1) One-dimensional case. For $n=1$, a Noetherian function is a restriction of a polynomial on a trajectory of a vector field $\partial / \partial x+\sum_{i} g_{i}(x, z) \partial / \partial z_{i}$ with polynomial coefficients, and the statement of Theorem 1 follows from [G3]. We recall here the arguments form [G3], because the proof in general case is based on the onedimensional case.

(2) Reduction to an integrable system. Union of all solutions of (2) for given polynomials $g_{i j}$ is an algebraic set. Complexity of this set is estimated.

(3) The Milnor fibers. For a one-parametric deformation of a (possibly non-isolated) intersection, we define the "Milnor fiber" $Z_{q}$ as follows: For a small nonzero value of parameter of the deformation, $Z_{q}$ consists of those points where the intersection is isolated and its multiplicity is at least $q$. Multiplicity of an isolated intersection equals the sum over $q$ of the Euler characteristics of $Z_{q}$.

(4) Maximal multiplicity in a generic family. We give an estimate for the maximal value of $q$ such that $Z_{q}$ is nonempty in a generic family. This allows to bound the number of nonzero terms in the formula for the multiplicity in terms of Euler characteristics, and the values of these nonzero terms.

\section{ONE-DIMENSIONAL CASE}

Noetherian chains in one-dimensional case are exactly trajectories of vector fields with polynomial coefficients, and Noetherian functions are polynomials restricted to trajectories of such vector fields.

Let $x \in \mathbb{C}, z=\left(z_{1}, \ldots, z_{m}\right) \in \mathbb{C}^{m}$, and let $\gamma=\{z=\psi(x)\}$ be a germ of a trajectory through $0 \in \mathbb{C}^{m+1}$ of a vector field $\xi=\partial / \partial x+\sum_{i} g_{i}(x, z) \partial / \partial z_{i}$, where $g_{i}$ are germs of analytic functions at $0 \in \mathbb{C}^{m+1}$. Let $P(t, z)$ be a germ of an analytic function at $0 \in \mathbb{C}^{m+1}$, and let $\phi(t)=P(t, \psi(t))$ be a restriction of $P(t, z)$ to $\gamma$. Suppose that $\phi(t) \not \equiv 0$, and let $\mu$ be the order of a zero of $\phi$ at $t=0$. Let $S(t, z, \epsilon)$ be a one-parametric deformation of $P$, i.e., a germ of an analytic function at $0 \in \mathbb{C}^{m+2}$ such that $S(t, z, 0)=P(t, z)$. We write $S_{\epsilon}(t, z)$ for $S(t, z, \epsilon)$ considered as a function in $\mathbb{C}^{m+1}$, with a fixed value of $\epsilon$.

Definition 2. For a positive integer $q$, the Milnor fiber $Z_{q}(\xi, S)$ of the deformation $S$ relative to the vector field $\xi$ is the intersection of a ball $\|(t, z)\| \leq \delta$ in $\mathbb{C}^{m+1}$ with a set $S_{\epsilon}=\xi S_{\epsilon}=\cdots=\xi^{q-1} S_{\epsilon}=0$, for a small positive $\delta$ and a complex nonzero $\epsilon$ much smaller than $\delta$. According to [Le], the homotopy type of $Z_{q}$ depends only on the deformation $S$ and on the vector field $\xi$. Let $\chi\left(Z_{q}\right)$ be the Euler characteristics of $Z_{q}$.

Theorem 2. Let $S$ be a one-parametric deformation of an analytic function $P$, and let $Z_{q}=Z_{q}(\xi, S)$ be the Milnor fibers of $S$ relative to an analytic vector field $\xi$. Suppose that $P$ restricted to a trajectory of $\xi$ through 0 has a zero of order $\mu<\infty$ at 0 . Let $Q=\max \left\{q: Z_{q} \neq \emptyset\right\}$. Then

$$
\mu=\sum_{q=1}^{Q} \chi\left(Z_{q}\right) .
$$


Proof. (See also [G3, Theorem 1].) Let $\left(t, y_{1}, \ldots, y_{m}\right)$ be a system of coordinates in $\mathbb{C}^{m+1}$ where $\xi=\partial / \partial t$, and let $\pi$ be projection $\mathbb{C}^{m+1} \rightarrow \mathbb{C}^{m}$ along the $t$-axis. Let $B_{r}$ be a closed ball of radius $r$ in $\mathbb{C}^{m}$ centered at the origin. We can choose a norm $\|\cdot\|$ in $\mathbb{C}^{m+1}$ so that $\{\|(t, y)\| \leq \delta\}=\left\{y \in B_{r},|t| \leq \delta\right\}$, where $r=r(\delta)$, and projection $\pi:\left\{S_{\epsilon}=0\right\} \rightarrow B_{r(\delta)}$ is a finite $\mu$-fold ramified covering (counting the multiplicities).

For a small $\delta>0$ and a small nonzero $\epsilon$ much smaller than $\delta$, projection $\pi: Z_{q} \rightarrow B_{r}$ is finite. For $y \in B_{r}$, a set $\pi^{-1} y \cap Z_{q}$ is finite. Hence its Euler characteristics $\zeta_{q}(y)=\chi\left(\pi^{-1} y \cap\right.$ $Z_{q}$ ) equals the number of points in it (not counting multiplicities). Then $\sum_{q=1}^{Q} \zeta_{q}(y) \equiv \mu$ does not depend on $y$. Standard "integration over Euler characteristic" arguments [V] show that

$$
\int_{B_{r}} \zeta_{q}(y) d \chi=\chi\left(Z_{q}\right), \quad \text { and } \quad \int_{B_{r}} \sum_{q=1}^{Q} \zeta_{q}(y) d \chi=\int_{B_{r}} \mu d \chi=\mu \text {. }
$$

This proves (3).

Lemma 1. Let $c_{0}, \ldots, c_{m}$ be a generic set of $m+1$ complex numbers. For a deformation $S(t, z, \epsilon)=P(t, z)+\epsilon \sum_{i=0}^{m} c_{i} t^{i}$, the sets $Z_{q}$ are nonsingular, for $q=1, \ldots, m+1$, and empty for $q>m+1$.

Proof. This is a special case of Thom's transversality theorem. See also [G3, Lemma 1].

Corollary. For the deformation in Lemma 1,

$$
\mu=\chi\left(Z_{1}\right)+\cdots+\chi\left(Z_{m+1}\right)
$$

Theorem 3. Let $\xi$ be a vector field defined by $d z_{i} / d t=g_{i}(t, z)$ where $g_{i}$ are polynomials of degree not exceeding $\alpha \geq 1$, and let $P$ be a polynomial of degree not exceeding $\beta \geq m$. Suppose that $P$ does not vanish identically on the trajectory $\gamma$ of $\xi$ through the origin. Then the multiplicity $\mu$ of a zero of $\left.P\right|_{\gamma}$ does not exceed

$$
\frac{1}{2} \sum_{k=0}^{m}[2 \beta+2 k(\alpha-1)]^{2 m+2}
$$

Proof. This follows from (4) and from an estimate $[\mathrm{M}]$ of the Euler characteristics of the set $Z_{q}$ defined by polynomial equations of degree not exceeding $\beta+(q-1)(\alpha-1)$.

\section{REDUCTION TO AN INTEGRABLE SYSTEM}

Let $x \in \mathbb{C}^{n}, z \in \mathbb{C}^{m}$, and let $g_{i j}(x, z)$ be analytic functions in $U \subset \mathbb{C}^{n+m}$.

Lemma 2. The union of all integral manifolds of (1) is an analytic subset of $U$.

Proof. Let $\xi_{i}, i=1, \ldots, n$ be the following vector fields tangent to the distribution (1):

$$
\xi_{i}=\frac{\partial}{\partial x_{i}}+\sum_{j=1}^{m} g_{i j}(x, z) \frac{\partial}{\partial z_{j}}
$$


For $c=\left(c_{1}, \ldots, c_{n}\right) \in \mathbb{C}^{n}$, let $\xi_{c}=c_{1} \xi_{1}+\cdots+c_{n} \xi_{n}$, and let $\gamma_{c}$ be a germ of a trajectory of $\xi_{c}$ through $\left(x_{0}, z_{0}\right)$. Let $\Lambda$ be the union of all $\gamma_{c}$. Then $\Lambda$ is a germ of an $n$-dimensional manifold.

If there exists an integral manifold of (1) through $\left(x_{0}, z_{0}\right)$, it contains all trajectories $\gamma_{c}$ and its germ at $\left(x_{0}, z_{0}\right)$ coincides with $\Lambda$. In this case, any bracket $\left[\xi_{j}, \xi_{k}\right]$ at each point of $\Lambda$ belongs to the subspace generated by $\xi_{i}$.

Due to $[\mathrm{G} 2$, Lemmas 4,5$]$, the opposite is also true: if any bracket $\left[\xi_{j}, \xi_{k}\right]$ at each point of $\Lambda$ belongs to the subspace generated by $\xi_{i}$, then $\Lambda$ is an integral manifold of (1).

Let $\Xi_{j k}=\left[\xi_{j}, \xi_{k}\right] \wedge \xi_{1} \cdots \wedge \xi_{n}$. If $\Lambda$ is an integral manifold of (1) then $\Xi_{j k}$ vanish at each point of each trajectory $\gamma_{c}$, for all $j$ and $k$. In particular,

$$
\xi_{c}^{\nu} \Xi_{j k}\left(x_{0}, z_{0}\right)
$$

the value at $\left(x_{0}, z_{0}\right)$ of the $\nu$ th derivative of $\Xi_{j k}$ along $\xi_{c}$, is zero for each $j, k$, and $\nu$. In the opposite case, when $\Xi_{j k}$ does not vanish identically on $\gamma_{c}$, for some $j, k$, and $c$, there exists a nonzero derivative (6).

Hence, $\left(x_{0}, z_{0}\right)$ belongs to an integral manifold of (1) iff all derivatives (6) vanish. As these derivatives are analytic in $\left(x_{0}, z_{0}\right)$, their common zeroes constitute an analytic set.

Theorem 4. Let $g_{i j}$ in (1) be polynomials of degree not exceeding $\alpha \geq 1$, and let $Y$ be the union of all integral manifolds of (1). Then $Y$ can be defined by a system of algebraic equations of degree not exceeding

$$
d_{Y}(m, n, \alpha)=\frac{(m+1)(\alpha-1)}{2}[2 \alpha(n+m+2)-2 m-2]^{2 m+2}+\alpha(n+2)-1 .
$$

Proof. From the proof of Lemma 2, the set $Y$ is defined by zeros of derivatives (6) of $\Xi_{i j}$ along $\xi_{c}$, for all $i, j$, and $c$. Here $\xi_{c}$ is a linear combination of the vector fields $\xi_{i}$ defined in $(5)$, and $\Xi_{j k}=\left[\xi_{j}, \xi_{k}\right] \wedge \xi_{1} \cdots \wedge \xi_{n}$.

As the coefficients of $\xi_{i}$ are polynomials of degree not exceeding $\alpha$, the coefficients of $\Xi_{j k}$ are polynomials of degree not exceeding $B=\alpha(n+2)-1$. If $\left(x_{0}, z_{0}\right)$ does not belong to $Y$, some of these coefficients do not vanish identically on the trajectory $\gamma_{c}$ of $\xi_{c}$ through $\left(x_{0}, z_{0}\right)$. Due to Theorem 3 , the multiplicity of zero at $\left(x_{0}, z_{0}\right)$ of these coefficients restricted to $\gamma_{c}$ cannot exceed

$$
N=\frac{1}{2} \sum_{k=0}^{m}[2 B+2 k(\alpha-1)]^{2 m+2}==\frac{1}{2} \sum_{k=0}^{m}[2 \alpha(n+k+2)-2 k-2]^{2 m+2} \text {. }
$$

This means that $Y$ can be defined by zeroes of derivatives (6) with $\nu \leq N$. But these derivatives are polynomials in $\left(x_{0}, z_{0}\right)$ of degree not exceeding $B+N(\alpha-1)$. Hence $Y$ is defined by a system of algebraic equations of degree not exceeding

$$
\frac{\alpha-1}{2} \sum_{k=0}^{m}[2 \alpha(n+k+2)-2 k-2]^{2 m+2}+\alpha(n+2)-1 .
$$

As $\alpha \geq 1$, this does not exceed the right side of (7). 


\section{The Milnor FiBers}

Let $g_{i j}(x, z)$ be germs of analytic functions at $0 \in \mathbb{C}^{n+m}$, and let $\{z=\psi(x)\}$ be a germ of an integral manifold of (1) through 0 . Let $P(x, z)=\left(P_{1}(x, z), \ldots, P_{n}(x, z)\right)$ be a germ of an analytic vector-function at 0 , and let $\phi_{i}(x)=P_{i}(x, \psi(x))$. Let $S(x, z, \epsilon)=$ $\left(S_{1}(x, z, \epsilon), \ldots, S_{m}(x, z, \epsilon)\right)$ be a one-parametric deformation of $P(x, z)$, i.e., a germ of an analytic vector-function at $0 \in \mathbb{C}^{n+m+1}$ such that $S(x, z, 0)=P(x, z)$. We write $S_{\epsilon}(x, z)$ for $S(x, z, \epsilon)$ considered as a function in $\mathbb{C}^{n+m}$, with a fixed value of $\epsilon$.

Let $Y$ be the union of all integral manifolds of (1). Due to Lemma 2, $Y$ is a germ of an analytic set. For $(x, z) \in Y$, let $\mu_{\epsilon}(x, z)$ be the multiplicity of the intersection $\left.S_{1, \epsilon}\right|_{\Lambda}=\cdots=\left.S_{n, \epsilon}\right|_{\Lambda}=0$ at $(x, z)$, where $\Lambda$ is an integral manifold of (1) through $(x, z)$.

Definition 3. For a positive integer $q$, the Milnor fiber $Z_{q}$ of the deformation $S$ relative to the distribution (1) is the intersection of a closed ball $B_{\delta}=\{\|(x, z)\| \leq \delta\}$ with a set of those points $(x, z) \in Y$ where $\mu_{\epsilon}(x, z) \geq q$, for a small positive $\delta$ and a complex nonzero $\epsilon$ much smaller than $\delta$. According to [Le] and Lemma 3 below, the homotopy type of $Z_{q}$ depends only on the deformation $S$, and on the coefficients $g_{i j}$ in (1). Let $\chi\left(Z_{q}\right)$ be the Euler characteristics of $Z_{q}$.

Lemma 3. Let $W_{q}$ be the set of small $(x, z, \epsilon) \in \mathbb{C}^{n+m+1}$ such that $(x, z) \in Y$, and $\mu_{\epsilon}(x, z) \geq q$. Then $W_{q}$ is a germ of an analytic set.

Proof. One can choose a system of coordinates $(x, y)$ in the neighborhood of $0 \in \mathbb{C}^{n+m}$ so that each integral manifold of (1) through a point $\left(x_{0}, y_{0}\right) \in Y$ is defined by $y=y_{0}$.

Due to $\left[\mathrm{AGV}\right.$, Lemma 5.5], the condition $\mu_{\epsilon}\left(x_{0}, y_{0}\right) \geq q$ depends only on the Taylor expansion $\check{S}_{i}$ in $x$ of $S_{i}$ at $\left(x_{0}, y_{0}, \epsilon\right)$ of order $q-1$. The coefficients of $\check{S}_{i}$ are

$$
\frac{\partial^{|\nu|} S_{i}}{\partial x^{\nu}}\left(x_{0}, y_{0}, \epsilon\right)
$$

which are analytic in $x_{0}, y_{0}, \epsilon$.

Let $K=\left(\begin{array}{c}q+n-1 \\ n\end{array}\right)$ be the number of monomials in $n$ variables of degree less than $q$. Consider $\check{S}_{i}$ as a vector in $\mathbb{C}^{K}$. For any multi-index $\nu=\left(\nu_{1}, \ldots, \nu_{n}\right)$, with $|\nu|=\nu_{1}+\cdots+$ $\nu_{n}<q$, consider $\left(x-x_{0}\right)^{\nu} \check{S}_{i}$ as a vector in $\mathbb{C}^{K}$, disregarding terms of order $q$ and higher in $x-x_{0}$.

Condition $\mu_{\epsilon}\left(x_{0}, y_{0}\right) \geq q$ means that rank of the set of $K n$ vectors $x^{\nu} \check{S}_{i}$ in $\mathbb{C}^{K}$ is at most $K-q$. This means vanishing of all $(K-q+1)$-minors of a $(K \times K n)$-matrix composed of these vectors. As the elements of this matrix are the partial derivatives (8), which are analytic in $\left(x_{0}, y_{0}, \epsilon\right)$, this, in combination with equations for $Y$, provides a system of analytic equations for $W_{q}$.

Theorem 5. Let $P=\left(P_{1}(x, z), \ldots, P_{n}(x, z)\right)$ be a germ of an analytic function at 0 in $\mathbb{C}^{n+m}$. Let $\psi=\left(\psi_{1}(x), \ldots, \psi_{m}(x)\right)$ be a germ of an analytic function at 0 in $\mathbb{C}^{n}$ satisfying $(2)$, and let $\phi_{i}(x)=P_{i}(x, \psi(x))$. Suppose that the intersection $\phi_{1}(x)=\cdots=\phi_{n}(x)=0$ is isolated at $x=0$, with the multiplicity $\mu$. Let $S(x, z, \epsilon)$ be a one-parametric deformation of $P$, let $Z_{q}$ be the Milnor fibers of $S$ relative to the distribution (1), and let $Q=\max \{q$ : 
$\left.Z_{q} \neq \emptyset\right\}$. Then

$$
\mu=\sum_{q=1}^{Q} \chi\left(Z_{q}\right)
$$

Proof. The arguments essentially repeat the arguments in the proof of Theorem 2, except an additional restriction to the set $Y$ of all integral manifolds of (1).

Let a system of coordinates $(x, y)$ in $\mathbb{C}^{n+m}$ be chosen, as in the proof of Lemma 3 , so that each integral manifold of (1) through $\left(x_{0}, y_{0}\right) \in Y$ is defined by $y=y_{0}$. Let $\pi$ be projection $\mathbb{C}^{n+m} \rightarrow \mathbb{C}^{n}$. Then $\pi:\left\{S_{\epsilon}=0\right\} \cap Y \rightarrow \pi Y$ is a finite $\mu$-fold ramified covering (counting the multiplicities). Let $\zeta_{q}(y)=\chi\left(\pi^{-1} y \cap Z_{q}\right)$ be the number of preimages of a point $y$ in $Z_{q}$, not counting multiplicities. Then $\sum_{q=1}^{Q} \zeta_{q}(y) \equiv \mu$ does not depend on $y$. We have

$$
\int_{\pi Y} \zeta_{q}(y) d \chi=\chi\left(Z_{q}\right), \quad \text { and } \quad \int_{\pi Y} \sum_{q=1}^{Q} \zeta_{q}(y) d \chi=\int_{\pi Y} \mu d \chi=\mu .
$$

The last equality holds because $\pi Y$ is a closed contractible set, hence its Euler characteristics equals 1.

Theorem 6. Let $g_{i j}$ in (1) be polynomials of degree not exceeding $\alpha$, and let $S_{i}$ be polynomials in $(x, z)$ of degree not exceeding $\beta$. Then the Milnor fiber $Z_{q}$ of $S$ can be defined by polynomial equations of degree not exceeding maximum of (7) and

$$
d(n, q, \alpha, \beta)=(K-q+1)[\beta+(q-1)(\alpha-1)], \quad \text { where } \quad K=\left(\begin{array}{c}
q+n-1 \\
n
\end{array}\right) .
$$

Proof. Let us fix a small nonzero $\epsilon$. According to the arguments in the proof of Lemma 3 , condition $\left(x_{0}, z_{0}\right) \in Z_{q}$ is equivalent to vanishing of all $(K-q+1)$-minors of a matrix composed of the partial derivatives (8) of $S_{\epsilon}$ of order $\nu<q$, in a system of coordinates $(x, y)$ where integral manifolds of $(1)$ are rectified.

Let us define a germ of the integral manifold $\Lambda$ of $(1)$ through $\left(x_{0}, z_{0}\right)$ by a function $\psi(x)$ satisfying (2). Equations (2) allow one to represent a partial derivative (8) as a polynomial in $\left(x_{0}, z_{0}\right)$ of degree not exceeding $\beta+\nu(\alpha-1)$. Hence the elements of our matrix are polynomials in $\left(x_{0}, z_{0}\right)$ of degree not exceeding $\beta+(q-1)(\alpha-1)$, and its $(K-q+1)$-minors are polynomials in $\left(x_{0}, z_{0}\right)$ of degree not exceeding $(K-q+1)[\beta+(q-1)(\alpha-1)$. These polynomials, in combination with equations for $Y$, provide a system of equations for $Z_{q}$.

Corollary. Under conditions of Theorem 6, the absolute value of the Euler characteristics of $Z_{q}$ does not exceed

$$
\frac{1}{2} \max \left(2 d_{Y}(m, n, \alpha), 2 d(n, q, \alpha, \beta)\right)^{2(m+n)},
$$

where $d_{Y}(m, n, \alpha)$ and $d(m, n, q)$ are defined in (7) and (10), respectively.

Proof. This follows from an estimate $[\mathrm{M}]$ of the Euler characteristics of the set $Z_{q}$ defined by polynomial equations of degree not exceeding maximum of (7) and (10). 


\section{MAXimal MUltiplicity IN A GENERIC FAMily}

Theorem 7. For an analytic mapping $P=\left(P_{1}, \ldots, P_{n}\right): \mathbb{C}^{n} \rightarrow \mathbb{C}^{n}$ and a nonnegative integer $r$, let

$$
P^{c}=\left(P_{1}^{c}, \ldots, P_{n}^{c}\right) \quad \text { where } P_{j}^{c}(x)=P_{j}(x)+\sum_{i:|i| \leq r} c_{i, j} x^{i} .
$$

Here $i=\left(i_{1}, \ldots, i_{n}\right)$ is a sequence of nonnegative integers, $|i|=i_{1}+\cdots+i_{n}$, and $x^{i}=$ $x_{1}^{i_{1}} \cdots x_{n}^{i_{n}}$.

For $0 \leq m \leq r$, the set of those $(x, c)$ where the multiplicity of $P^{c}$ at $x$ exceeds

$$
Q(m, n)=\left(\frac{n+m}{1+\cdots+1 / n}\right)^{1+\cdots+1 / n} \prod_{k=1}^{n}\left(\frac{(k-1) !}{k}\right)^{1 / k}
$$

has codimension greater than $n+m$.

Corollary. Let $P_{z}(x)=\left(P_{z, 1}(x), \ldots, P_{z, n}(x)\right)$ be a generic family of analytic mappings $\mathbb{C}^{n} \rightarrow \mathbb{C}^{n}$ depending on parameters $z \in \mathbb{C}^{m}$. Then the multiplicity of $P_{z}$, at any point $x \in \mathbb{C}^{n}$ and for any $z$, is less than (13).

To prove Theorem 7 we need the following

Definition 4. For an analytic set $V$ of codimension $r$, we define the order $\left.\operatorname{ord}_{x_{0}} P\right|_{V}$ of an analytic function $P$ on the set $V$ at $x_{0} \in V$ as a maximal integer $\nu$ such that, for a generic $(\mathrm{r}+1)$-dimensional plane $L, P(x)=o\left(\left|x-x_{0}\right|^{\nu-1}\right)$, for all $x$ in at least one of irreducible components of $V \cap L$. If such a number does not exist (i.e., when $P$ vanishes identically on an irreducible component of $V$ through $x_{0}$ ) we define $\left.\operatorname{ord}_{x_{0}} P\right|_{V}=\infty$. For $V=\mathbb{C}^{n},\left.\quad \operatorname{ord}_{x_{0}} P\right|_{V}=\operatorname{ord}_{x_{0}} f$ is the usual vanishing order of $P$ at $x_{0}$.

For example, let $V=\left\{x_{1}^{2}=x_{2}^{3}\right\}$ and $x_{0}=(0,0)$. Then $\left.\operatorname{ord}_{0} x_{1}\right|_{V}=2$ and $\left.\operatorname{ord}_{0} x_{2}\right|_{V}=1$.

Lemma 4. Let $r \geq 0$, and let $P^{c}$ be defined as in (12). For a sequence $\nu_{1} \leq \cdots \leq \nu_{n} \leq r$ of nonnegative integers, let $X_{\nu_{1}, \ldots, \nu_{n}}$ be the set of $(x, c)$ where

$$
\begin{aligned}
& \operatorname{ord}_{x} P_{1}^{c}=\nu_{1},\left.\quad \operatorname{ord}_{x} P_{2}^{c}\right|_{P_{1}^{c}=0}=\nu_{2}, \ldots,\left.\operatorname{ord}_{x} P_{n}^{c}\right|_{P_{1}^{c}=\cdots=P_{n-1}^{c}=0}=\nu_{n} ; \\
& \quad \operatorname{ord}_{x} P_{j}^{c} \geq \nu_{1}, \text { for } j>1,\left.\operatorname{ord}_{x} P_{j}^{c}\right|_{P_{1}^{c}=0} \geq \nu_{2}, \text { for } j>2, \ldots, \\
& \left.\quad \operatorname{ord}_{x} P_{n}^{c}\right|_{P_{1}^{c}=\cdots=P_{n-2}^{c}=0} \geq \nu_{n-1} .
\end{aligned}
$$

The codimension of $X_{\nu_{1}, \ldots, \nu_{n}}$ is not less than

$$
\nu_{1}\left(\begin{array}{c}
\nu_{1}+n-2 \\
n-1
\end{array}\right)+\nu_{2}\left(\begin{array}{c}
\nu_{2}+n-3 \\
n-2
\end{array}\right)+\cdots+\nu_{n}
$$

and the multiplicity of $P^{c}$ at any point of $X_{\nu_{1}, \ldots, \nu_{n}}$ is less than $\nu_{1} \cdots \nu_{n}$.

Proof. The condition $\operatorname{ord}_{x} P_{j}^{c} \geq \nu_{1}$, for all $j$, means that the values of $-c_{i, j}$, for $|i|<\nu_{1}$, coincide with the coefficients of the Taylor expansion of $P_{j}$ at $x$ of the order $\nu_{1}-1$. This gives $n\left(\begin{array}{c}\nu_{1}+n-1 \\ n\end{array}\right)$ independent conditions on $c_{i, j}$. 
Let us fix $P_{1}^{c}$ and consider a one-dimensional linear subspace $l$ outside the tangent cone to $\left\{P_{1}^{c}=0\right\}$ at $x$. We can choose coordinates so that $l$ is the $x_{n}$-axis. Let us fix all the terms in $P_{j}^{c}$, for $j>1$, except those that do not contain $x_{n}$. The condition $\left.\operatorname{ord}_{x} P_{j}^{c}\right|_{P_{1}^{c}=0} \geq \nu_{2}$, for $j>1$, defines at most one possible value for each of the remaining terms. This gives us at least $(n-1)\left[\left(\begin{array}{c}\nu_{2}+n-2 \\ n-1\end{array}\right)-\left(\begin{array}{c}\nu_{1}+n-2 \\ n-1\end{array}\right)\right]$ additional independent conditions on $c_{i, j}$. The same arguments allow one to prove that the codimension of $X_{\nu_{1}, \ldots, \nu_{d}}$ is not less than

$$
n\left(\begin{array}{c}
\nu_{1}+n-1 \\
n
\end{array}\right)+(n-1)\left[\left(\begin{array}{c}
\nu_{2}+n-2 \\
n-1
\end{array}\right)-\left(\begin{array}{c}
\nu_{1}+n-2 \\
n-1
\end{array}\right)\right]+\cdots+\left[\left(\begin{array}{c}
\nu_{n} \\
1
\end{array}\right)-\left(\begin{array}{c}
\nu_{n-1} \\
1
\end{array}\right)\right] .
$$

Applying the identity $k\left(\begin{array}{c}\nu_{n-k+1}+k-1 \\ k\end{array}\right)-(k-1)\left(\begin{array}{c}\nu_{n-k+1}+k-2 \\ k-1\end{array}\right)=\nu_{n-k+1}\left(\begin{array}{c}\nu_{n-k+1}+k-2 \\ k-1\end{array}\right)$, we obtain (14).

To estimate the multiplicity $\mu$ of $f_{c}$ at a point $x \in X_{\nu_{1}, \ldots, \nu_{n}}$, we have to count the number of zeros (with their multiplicities) of a system of equations $P_{1}^{c}=\cdots=P_{n-1}^{c}=P_{n}^{c}-\epsilon=0$ converging to $x$ as $\epsilon \rightarrow 0$. Due to the condition $\left.\operatorname{ord}_{x} P_{n}^{c}\right|_{P_{1}^{c}=\cdots=P_{n-1}^{c}=0}=\nu_{n}$, this number is less than $\nu_{n}$ multiplied by the multiplicity $\mu^{\prime}$ of $\left\{P_{1}^{c}=\cdots=P_{n-1}^{c}=s=0\right\}$ at $x$, where $s$ is a generic linear function. The same arguments show that $\mu^{\prime}$ is less than $\nu_{n-1}$ multiplied by the multiplicity of $\left\{P_{1}^{c}=\cdots=P_{n-2}^{c}=s_{1}=s_{2}=0\right\}$ at $x$, where $s_{1}$ and $s_{2}$ are generic linear functions. Repeating these arguments, we obtain the necessary estimate $\mu<\nu_{1} \cdots \nu_{n}$

Proof of Theorem 7. Due to Lemma 4, we have to estimate maximal possible value of $\nu_{1} \cdots \nu_{n}$ over the sequences $\left(\nu_{1}, \ldots, \nu_{n}\right)$ such that (14) does not exceed $n+r$. Replacing $\left(\begin{array}{c}\nu_{n-k+1}+k-2 \\ k-1\end{array}\right)$ by $\nu_{n-k+1}^{k-1} / k-1$ !, we see that (14) is not less than $\sum_{k=1}^{n} \nu_{n-k+1}^{k} /(k-1)$ !. Thus it is enough to maximize $\nu_{1} \cdots \nu_{n}$ when $\sum_{k=1}^{n} \nu_{n-k+1}^{k} /(k-1) !=n+r$. Substituting $u_{k}=\nu_{n-k+1}^{k}$, we have to maximize $\prod_{k=1}^{n} u_{k}^{1 / k}$ when $\sum_{k=1}^{n} u_{k} /(k-1) !=n+r$. The maximum (13) is achieved when

$$
u_{k}=\frac{(k-1) !(n+r)}{k(1+\cdots+1 / n)}
$$

\section{Proof of Theorem 1}

Consider a deformation $S(x, z, \epsilon)$ of the polynomial $P(x, z)$ defined by

$$
S_{j}(x, z, \epsilon)=P_{j}(x, z)+\epsilon \sum_{i:|i| \leq m} c_{i, j} x^{i}
$$

where $c_{i, j}$ are generic complex numbers. From Theorem 7 , the Milnor fibers $Z_{q}$ of this deformation are empty for $q \geq Q(m, n)$, where $Q(m, n)$ is defined in (13).

According to (9),

$$
\mu=\sum_{q=1}^{Q(m, n)} \chi\left(Z_{q}\right) \leq Q(m, n) \max _{q \leq Q(m, n)}\left|\chi\left(Z_{q}\right)\right|
$$


From (11), the right side of (15) does not exceed

$$
\frac{1}{2} Q(m, n) \max \left(2 d_{Y}(m, n, \alpha), 2 d(n, Q(m, n), \alpha, \beta)\right)^{2(m+n)},
$$

where $d_{Y}$ and $d$ are defined in (7) and (10), respectively. The value of $d(n, q, \alpha, \beta)$ in (10) does not exceed

$$
(q+n-1)^{n}(\beta+(q-1)(\alpha-1))<(q+n)^{n}(\beta+q(\alpha-1)) .
$$

The statement of Theorem 1 follows now from the following estimate for $Q(m, n)$ :

Proposition 1. The value of $Q(m, n)$ in (13) does not exceed

$$
e n\left(\frac{e(n+m)}{\sqrt{n}}\right)^{\ln n+1}\left(\frac{n}{e^{2}}\right)^{n} .
$$

Proof. The statement is obvious for $n=1$.

Let $n>1$. From the obvious inequality

$$
\frac{3}{2} \leq 1+\cdots+\frac{1}{n} \leq \ln n+1
$$

we have

$$
Q(m, n) \leq \frac{2}{3}(n+m)^{\ln n+1} \prod_{k=1}^{n}\left(\frac{(k-1) !}{k}\right)^{1 / k} .
$$

Next, we apply

$$
k ! \leq e \sqrt{k}\left(\frac{k}{e}\right)^{k}
$$

to obtain

$$
Q(m, n)<\frac{2}{3}(n+m)^{\ln n+1} e^{1+\cdots+\frac{1}{n}} n ! e^{-n} \prod_{k=1}^{n} k^{-\frac{3}{2 k}} .
$$

The inequality (18) follows from the inequality

$$
\ln (k !)-\frac{\ln k}{2}=\ln 1+\cdots+\ln k-\frac{\ln k}{2} \leq \int_{1}^{k} \ln x d x=n \ln k-k+1 .
$$

Applying once more (17) and (18), we obtain

$$
Q(m, n)<\frac{2}{3} e \sqrt{n}(e(n+m))^{\ln n+1}\left(\frac{n}{e^{2}}\right)^{n} \prod_{k=1}^{n} k^{-\frac{3}{2 k}} .
$$


To estimate the product in the right side of (19), we apply the inequality

$$
\sum_{k=1}^{n} \frac{\ln k}{k}>\frac{\ln ^{2} n}{2}-c, \quad \text { where } \quad c=\frac{\ln ^{2} 5}{2}-\sum_{k=1}^{4} \frac{\ln k}{k}-\frac{\ln 5}{10}<0.075
$$

To prove $(20)$, we consider $(\ln x) / x$, which is concave for $x>e^{3 / 2} \approx 4.48$. Hence, for $n \geq 5$,

$$
\sum_{k=5}^{n} \frac{\ln k}{k}-\frac{\ln 5}{10}-\frac{\ln n}{2 n}>\int_{5}^{n} \frac{\ln x}{x} d x=\frac{\ln ^{2} n-\ln ^{2} 5}{2} .
$$

This implies (20) for $n \geq 5$, and it can be easily verified for $n=2,3,4$. Exponentiating (20), we have

$$
\prod_{k=1}^{n} k^{-\frac{3}{2 k}}<e^{3 c / 2} n^{-(\ln n) / 2}<\frac{3}{2} n^{-(\ln n) / 2} .
$$

Substituting this into (19), we obtain (16).

\section{REFERENCES}

[AGV] V.I. Arnold, S.M. Gusein-Zade, A.N. Varchenko, Singularities of Differentiable Maps, Volume I, Birkhäuser, Basel, 1986.

[G1] A. Gabrielov, Multiplicities of Pfaffian Intersections and the Lojasiewicz Inequality, Selecta Mathematica, New Series 1 (1995), 113-127.

[G2] A. Gabrielov, Multiplicities of zeros of polynomials on trajectories of polynomial vector fields and bounds on degree of nonholonomy, Math. Research Letters 2 (1995), 437-451.

[G3] A. Gabrielov, Multiplicity of a zero of an analytic function on a trajectory of a vector field, Preprint (1997).

[Kh1] A.G. Khovanskii, On a class of systems of transcendental equations, Soviet Math. Dokl 22 (1980), $762-765$.

[Kh2] A.G. Khovanskii, Fewnomials, AMS Translation of mathematical monographs, vol. 88, AMS, Providence, RI, 1991.

[Kh3] A.G. Khovanskii, Malochleny, In Russian, FAZIS, Moscow, 1996.

[Le] Lê Dũng Tráng, Some Remarks on Relative Monodromy, Real and Complex Singularities, Oslo 1976 (P. Holm, ed.), Sijthoff \& Noordhoff International Publishers, 1977, pp. 397-403.

[M] J. Milnor, On the Betti Numbers of Real Varieties, Proc. AMS 15 (1964), 275-280.

[N] Y.V. Nesterenko, Estimates for the number of zeros of certain functions, New Advances in Transcendence Theory (A. Baker, eds.), Proc. Conf. Durham 1986, Cambridge Univ. Press, 1988, pp. 263-269.

[R] J.-J. Risler, A bound for the degree of nonholonomy in the plane, Theoret. Comput. Sci. 157 (1996), 129-136.

[T] J.-C. Tougeron,, Algèbres analytiques topologiquement nœethériennes, Théorie de Hovanskii, Ann. Inst. Fourier 41 (1991), 823-840.

[V] O. Viro, Some integral calculus based on Euler characteristic, Topology and geometry-Rohlin Seminar, Lecture Notes in Math., vol. 1346, Springer, Berlin-New York, 1988, pp. 127-138.

[W] M. Waldschmidt, Sur la nature arithmétique des valeurs de fonctions modulaires, Séminaire Bourbaki 1996-97, Exposé 824, ENS, Paris, 1997.

Department of Mathematics, Purdue University, W. Lafayette, IN 47907-1395, USA 
E-mail address: agabriel@math.purdue.edu

Department of Mathematics, Independent University, Moscow, Russia

Department of Mathematics, University of Toronto, Toronto, Canada M5S 1A1

E-mail address: askold@math.toronto.edu 